\title{
Über die Autoren/Abstracts
}

\section{Martina Nied Curcio}

Studium der Germanistik/DaF und Romanistik in Karlsruhe und Konstanz (Dr. phil. in Italienischer Sprachwissenschaft). Nach DAAD-Lektorat und diversen Lehraufträgen seit 2005 Professorin an der Universität Roma Tre. E-Mail: nied@uniroma3.it

\section{Daniel Jaeger}

Studium der Germanistik und Philosophie sowie DaF in Berlin und Ithaca, NY (M.A.). DAAD-Lektor in Neapel und Rom (Universität >La Sapienza<). Seit 2006 wieder in Berlin. E-Mail: jaeger.dan@web.de

\section{Almut Meyer}

Lic. phil.; Studium der Germanistik sowie Musik und Musikwissenschaft in Deutschland und Finnland; 1994-2000 Forschungsstipendien und Lehraufträge an den Universitäten Jyväskylä und Turku; 2000 Lizenziatenarbeit zum Thema Musikkulturen im DaF-Unterricht. Seit 2002 Lektorin für Deutsch (Fachsprache Recht) an der Rechtswissenschaftlichen Fakultät der Universität Turku/Finnland. Arbeitsschwerpunkte: Fachsprache Recht und Curriculumentwicklung.

\section{Eva Neuland}

Professorin für Germanistik, Didaktik der deutschen Sprache und Literatur an der Bergischen Universität Wuppertal. Arbeitsschwerpunkte: Deutsche Gegenwartssprache, Soziolinguistik, Pragmalinguistik, Gesprächsforschung, Jugendsprachforschung, Sprachdidaktik, Deutsch als Fremdsprache.

\section{Markus Raith}

Dr. phil.; Studium der Germanistik und Romanistik in Stuttgart, Montpellier und Bordeaux; Referendariat; derzeit DAADLektor für deutsche Sprache, Literatur und Landeskunde an der Universität Poitiers/Frankreich. Arbeitsgebiete: Mediendidaktik, kulturwissenschaftliche Landeskunde im DaF-Unterricht (insbesondere Fremd- und Selbstbilder, Kulturtransfer), deutsche Literatur des 19. und 20. Jahrhunderts im europäischen Kontext und ihre Didaktik.

\section{Wanna Saengaramruang}

Assoc. Prof. Dr. phil.; Studium der Fächer Deutsch, Geschichte und Bibliothekswissenschaft an der Chulalongkorn Universität. 1979 Lehrstuhl für Deutsch bzw. Germanistik an der Deutschen Abteilung der Chulalongkorn Universität, Bangkok. 1992 Promotion bei Gerhard Neuner an der Universität Kassel, Fachgebiet Deutsch als Fremdsprache. Seit 2000 Leiterin der Deutschen Abteilung der Chulalongkorn Universität und seit 2007 Präsidentin des Thailändischen Deutschlehrerverbandes. Forschungsgebiete: Deutsch als Tertiärsprache nach Englisch in Thailand, Lernergrammatik/Deutschlehrwerk für thailändische Deutschlernende; Thai-Deutsche Wörterbücher, Übersetzungswissenschaft der Sprachenpaare Deutsch/Thai.

\section{Lisa Schlanstein}

Studium der Germanistik und Geschichte in Freiburg (M. A.). Seit 1988 Lektorin an der Universität > La Sapienza< in Rom (Facoltà di Scienze Umanistiche). Seit 1990 Mitarbeiterin am Goethe-Institut Rom. E-Mail: 1schlanstein@libero.it 
Karin Schmidt

DAAD-Lektorin Usbekistan 1995-2001; seit 2001 Wiss. Mitarbeiterin am Studiengebiet Deutsch als Fremdsprache der FU Berlin; seit 2004/05 Koordinatorin der AG »Erinnerungsorte im DaF-Unterricht«. Weitere Arbeitsschwerpunkte: Grammatikvermittlung; Schreibentwicklung; Lernerkorpus »Falko" (http:// www2.huberlin.de/korpling/projekte/ falko/).

\section{Sabine Schmidt}

Dr. phil.; DAAD-Lektorin Polen 19911995; 1998-2001 Freie Mitarbeiterin am Deutschen Kulturinstitut/Goethe-Institut Tallinn; seit 2002 Mitarbeiterin am
Sprachenzentrum der FU Berlin, Bereich DaF; seit 2004/05 Koordinatorin der AG »Erinnerungsorte im DaF-Unterricht«.

\section{Jianpei Yang}

M. A., geb. 1975; 1994-1998 Studium der Germanistik an der Fremdsprachenhochschule Sichuan; 1998-1999 Dolmetscher und Übersetzer bei der deutschen Firma Happy Arts \& Crafts (Ningbo) Co. Ltd.; 1999-2002 Magisterstudium an der Fremdsprachenhochschule Xi'an; seit 2002 DaF-Dozent am Deutsch-Kolleg der Tongji-Universität; seit 2004 Promotion im Fach Deutsch als Fremdsprache an der TU Berlin. 


\begin{tabular}{|c|c|c|c|c|c|}
\hline 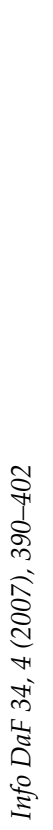 & 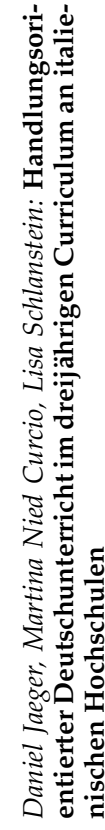 & 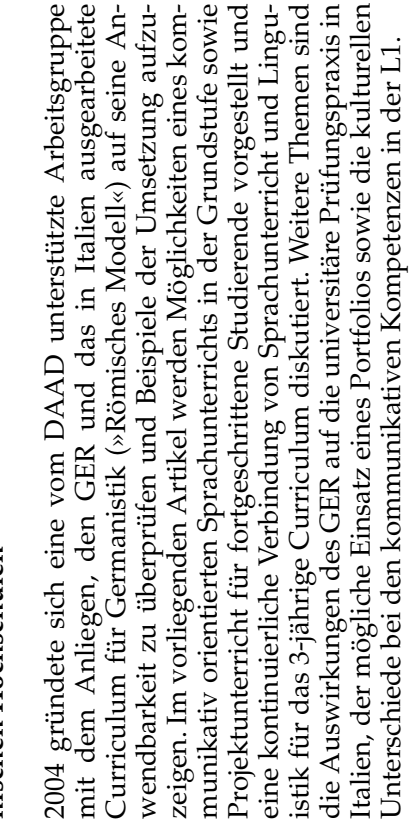 & 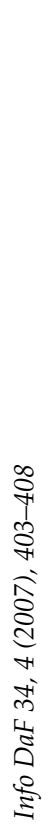 & 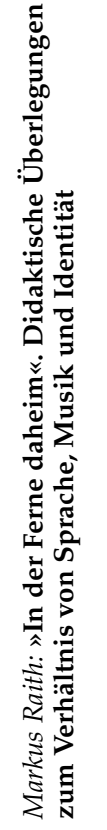 & 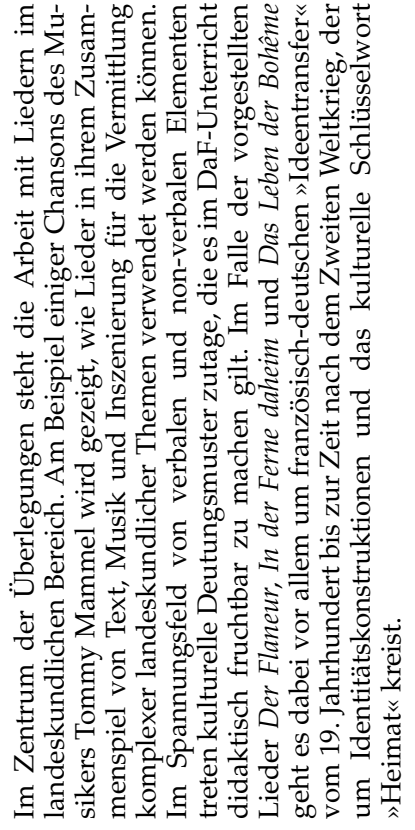 \\
\hline 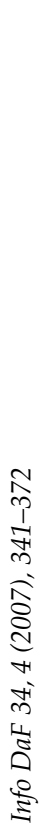 & 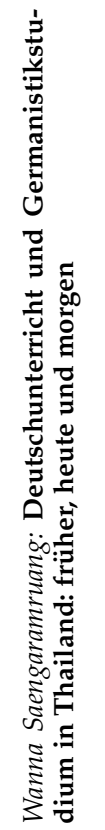 & 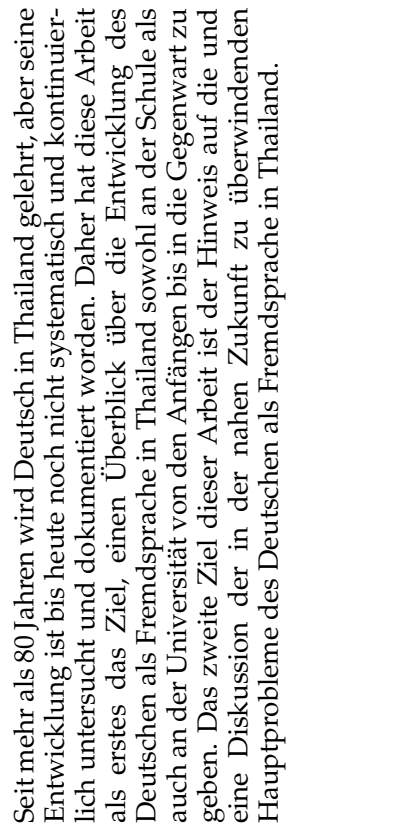 & 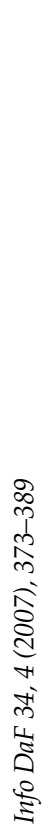 & 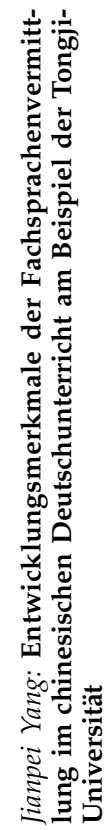 & 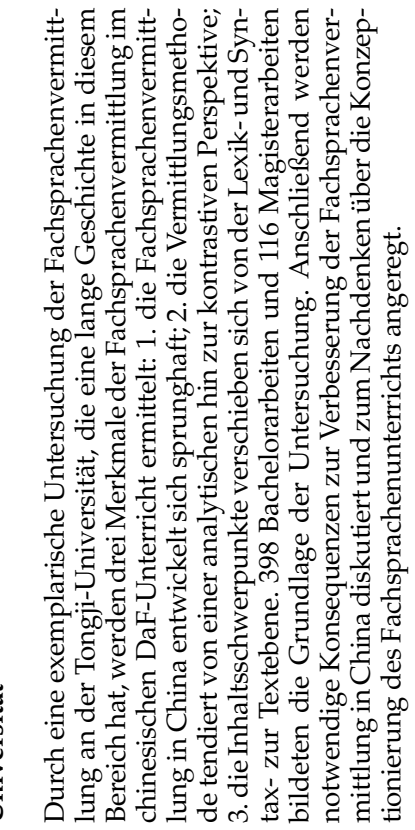 \\
\hline
\end{tabular}




\begin{tabular}{|c|c|c|c|c|}
\hline 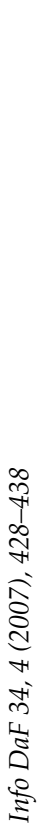 & 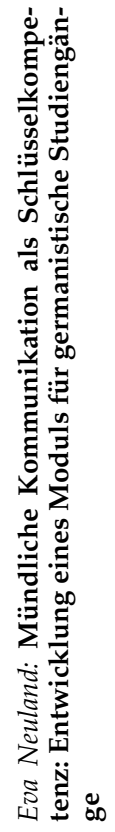 & 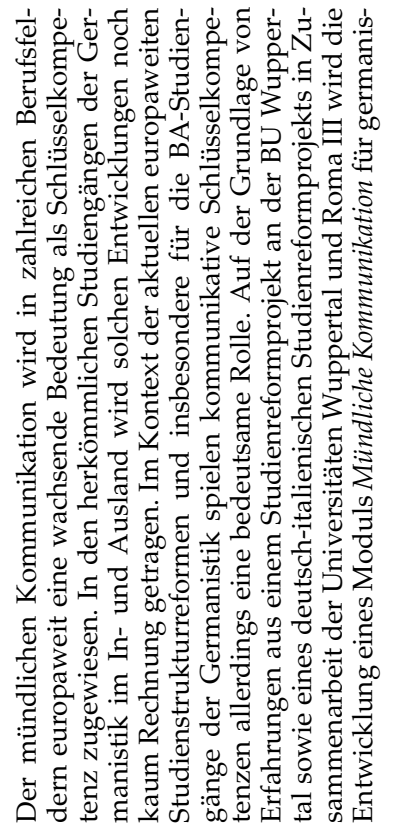 & 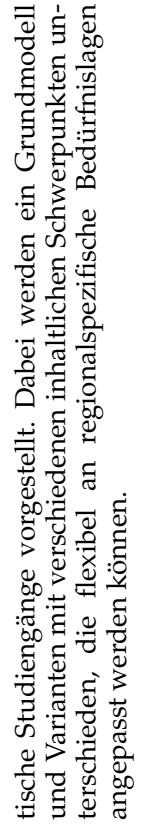 & \\
\hline 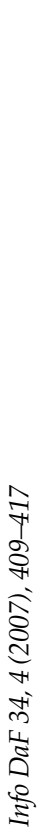 & 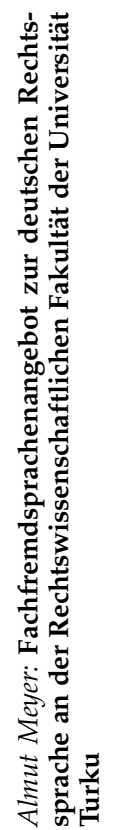 & 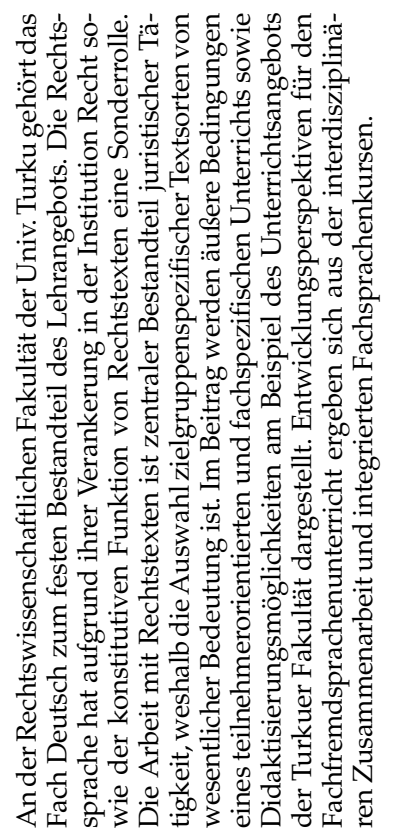 & 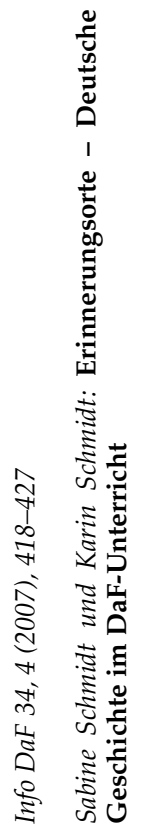 & 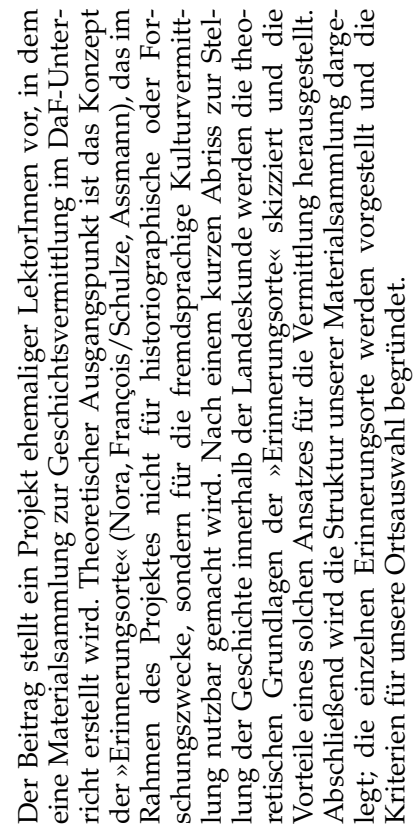 \\
\hline
\end{tabular}

